Journal of The Magnetics Society of Japan Vol. 13, Supplement, No. S1 (1989)

(C) 1989 by The Magnetics Society of Japan

\title{
VECTOR MAGNETIZATION OF RECORDING MEDIA
}

Dennis SPELIOTIS, David BONO, and Patrick JUDGE

Digital Measurement Systems, Inc., 8 Ray Avenue, Burlington, MA 01803, USA

\begin{abstract}
Advanced magnetic recording media may be oriented with a preferred direction of magnetization in or out of the plane. The angular dependence of the magnetic properties of the media provides important
\end{abstract} information for determining the mechanism of magnetization reversal and for analyzing the recording process, since the head field incorporates parallel and perpendicular components in its interaction with the recording media. To obtain the intrinsic magnetic properties, it is necessary to compensate the demagnetizing fields, and for this purpose, we have added orthogonal biaxial detecting coils to our VSM for the simultaneous measurement of the vector magnetization components. In this paper, we report on vector magnetization measurements on most of the commonly used magnetic recording media, including SVHS, $8 \mathrm{~mm}$ video, $\mathrm{CrO}_{2}, \mathrm{Ba}$-ferrite, $\mathrm{Co}-\mathrm{Ni}-\mathrm{P}$ thin film, and $\mathrm{CoCr}$.

\section{INTRODUCTION}

Magnetic recording media are typically in the form of thin films or thin coatings of particulate assemblies. The anisotropy of the individual particles or crystallites may be oriented in the plane, out of the plane, or random. A very fundamental question pertaining to these media is the mechanism of magnetization reversal, and one way to experimentally investigate it is by measuring the angular dependence of the magnetic properties of the media $[1,2]$. For media oriented in the plane, it is quite simple to measure the angular dependence of the magnetic properties in the plane, since we do not have to contend with any demagnetizing fields. The field of a typical ring-type recording head, however, changes from purely horizontal over the center of the gap to purely perpendicular far away from the gap edge. Furthermore, many advanced media, such as $\mathrm{CoCr}$ perpendicular anisotropy media [3] and $\mathrm{Ba}$-ferrite particulate media [4], may have a preferred direction of magnetization perpendicular to the media plane. In such cases, the measurement of the intrinsic magnetic properties out of the media plane is obscured by the demagnetizing fields which arise on account of the sample shape when the external field is applied at any angle away from the plane. In this respect, it is particularly useful to measure the vector magnetization [5] in order to determine the demagnetizing field, and thus provide the appropriate corrections to the applied field in order to obtain the correct amplitude and direction of the effective applied field.

To perform the vector magnetization measurements, we have added vector (two-dimensional) capability to the DMS Model 880 VSM, which also incorporates computercontrolled angular sample positioning. The vector magnetization is measured by orthogonal pairs of pickup coils, one along $x$ (the direction of the applied field $\mathrm{H}_{a}$ ) and the other along $y$. In this magnetometer, a field feedback loop permits the precise digital setting of the field before the magnetization is measured. Therefore, both the $M_{\mathbf{X}}$ and $\mathrm{M}_{\mathrm{y}}$ components of the magnetization can be measured successively for each field setting using the same signal processor.

In this paper, we report on the vector magnetization of various recording media, both thin film and 
particulate, with different anisotropies and orientations.

\section{EXPERIMENTAL SYSTEM AND ANALYSIS}

The vector coil arrangement for the biaxial VSM is shown schematically in Figure 1, along with the various angles for the magnetization $M$, the applied field $\mathrm{H}_{\mathrm{a}}$ (assumed along the $x$ axis), and the effective field $\mathrm{H}_{e}$ (corrected for the demagnetizing field). The sample is shown in a vertical plane, so the applied field rotates out of the plane of the sample.

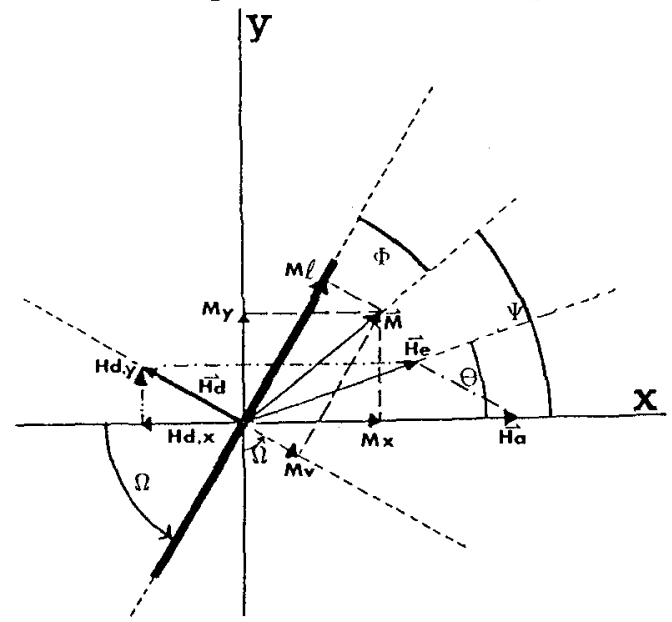

Fig. 1 Schematic diagram of the vector quantities and angles shown as a top view of biaxial (xy) VSM.

The other case, where the sample plane is horizontal (xy), is also of interest for samples that are oriented in the plane, but is quite simple to analyze since there are no corrections required (there are no demagnetizing fields). The basic relations can be easily derived from Figure 1, which shows the sample plane rotated by an angle $\Omega$ from the $x$ axis.

The magnetization $\overrightarrow{\mathrm{M}}$ makes an angle $\psi$ with the $x$ axis and an angle $\phi$ with the sample plane. Therefore,

$$
\begin{aligned}
& \psi=\tan ^{-1}\left(M_{y} / M_{x}\right) \text { and } \phi=\Omega-\psi \\
& \text { and } M=|\vec{M}|=\left(M_{x}{ }^{2}+M_{y}{ }^{2}\right) 1 / 2
\end{aligned}
$$

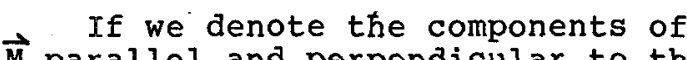
$\vec{M}$ parallel and perpendicular to the sample plane as $\mathrm{M}_{1}$ and $\mathrm{M}_{\mathrm{V}}$, respectively, we have:

$$
M_{I}=M \cos \phi \text { and } M_{V}=M \sin \phi \text {. }
$$

Therefore, the demagnetizing field $\mathrm{H}_{\mathrm{d}}$ can be determined from:

$$
\begin{gathered}
H_{d}=\mid \begin{array}{ccc}
\left|\vec{H}_{d}\right| & =4 \pi N \quad M_{v} \\
4 \pi N & M & \sin \phi
\end{array}=
\end{gathered}
$$

where $4 \pi \mathrm{N}$ is the demagnetizing factor.

Consequently, the effective field $\mathrm{H}_{e}$ can be determined from the expression:

$$
\begin{gathered}
\mathrm{H}_{e}=\left|\underset{\mathrm{H}_{e}}{ }\right|=\left|\overrightarrow{\mathrm{H}}_{\mathrm{d}}+\overrightarrow{\mathrm{H}}_{\mathrm{a}}\right|= \\
\left(\mathrm{H}_{\mathrm{e}, \mathrm{x}}{ }^{2}+\mathrm{H}_{\mathrm{e}, \mathrm{y}}{ }^{2}\right) \mathrm{l} / 2
\end{gathered}
$$

where

$\mathrm{H}_{\mathrm{e}, \mathrm{x}}=\mathrm{H}_{\mathrm{a}}-4 \pi \mathrm{N} \cdot \mathrm{M} \cdot \sin \phi \cdot \sin \Omega$

$\mathrm{H}_{\mathrm{e}, \mathrm{Y}}=4 \pi \mathrm{N} \cdot \mathrm{M} \cdot \sin \phi \cdot \cos \Omega$.

The effective field $\overrightarrow{\mathrm{H}}_{e}$ is at

an angle $\theta$ with respect to the $x$ axis, where $\theta=\tan ^{-1}\left(\mathrm{H}_{e}, y\right)$ $\mathrm{H}_{\mathrm{e}, \mathrm{x}}$ ).

We may also easily obtain the components of $\mathrm{H}_{e}$ parallel and perpendicular to the sample plane:

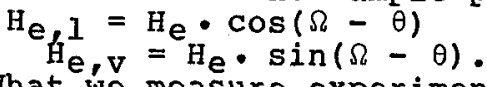

What we measure experimentally is $M_{X}\left(H_{a}\right)$ and $M_{y}\left(H_{a}\right)$ at various values of $\Omega$. To obtain the intrinsic $\mathrm{M}-\mathrm{H}$ loops without the demagnetizing field, we may use the above expressions to obtain $M_{X}\left(H_{e}, x\right)$ and $M_{y}\left(H_{e}, x\right)$ at various values of $\Omega$. As was pointed out by Ouchi and Iwasaki [5], in the $\mathrm{M}_{X}\left(\mathrm{H}_{\mathrm{a}}\right)$ curve at $\mathrm{H}_{\mathrm{a}}=\mathrm{H}_{\mathrm{C}}$, we have $\mathrm{M}_{\mathrm{X}}=0$, but $M_{Y} \neq 0$, except at $\Omega=0$ ó and $\Omega=900$, and the demagnetizing field reflects on the $\mathrm{M}_{X}\left(H_{e}, x\right)$ curve, resulting in a different $\mathrm{H}_{\mathrm{C}}(\Omega)$. It it this intrinsic $\mathrm{H}_{C}(\Omega)$ curve that is of importance in determining the mechanism of magnetization reversal. 
Using the above equations, we can also obtain the following relationships:

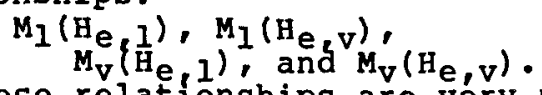

These relationships are very useful in analyzing the fundamental vector magnetization and field interactions in the recording process. Finally, as again was shown by Ouchi and Iwasaki [5], it is possible from the vector magnetization data to obtain the intrinsic torque curve corrected for the effect of demagnetization, and thus be able to determine the anisotropy constant of the media:

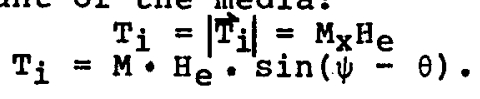

\section{RESULTS AND DISCUSSION}

We measured the vector magnetization in the plane and out of the plane for various media of current interest, including longitudinally oriented particulate media (SVHS, $\mathrm{CrO}_{2}$, and metal particle (MP)), non-oriented and perpendicularly oriented Ba-ferrite (BF) media, and longitudinal and perpendicular thin film media (CO-Ni-P and $\mathrm{CoCr}$ ). Since the amount of data obtained could not be presented within the limited size of this paper, we decided to present some important general vector results for all of these media and leave the further analysis and interpretation to a future publication.

We selected an angle $\Omega=60^{\circ}$ from the plane for comparing the uncorrected $\left(\mathrm{M}-\mathrm{H}_{\mathrm{a}}\right)$ and corrected $\left(\mathrm{M}-\mathrm{H}_{\mathrm{e}}\right)$ loops of the various media. Figures 2-4 show the results for SVHS, $\mathrm{CrO}_{2}$, and $\mathrm{MP}$ ( $8 \mathrm{~mm}$ video) tapes. Clearly, at $\mathrm{H}_{\mathrm{a}}=\mathrm{H}_{\mathrm{C}}$, there is a very large $M_{y}$ component, resulting in a large correction for the intrinsic $\mathrm{M}-\mathrm{H}_{e}$ curves. The Co-Ni-P thin film media exhibit similar behavior, as shown in Figure 5 .
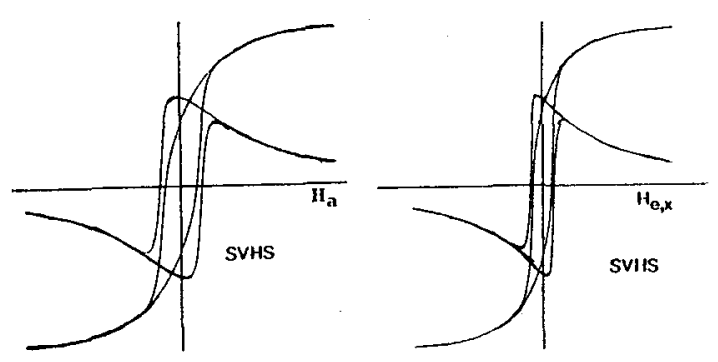

Fig. $2 \quad \mathrm{M}_{X}-\mathrm{H}_{a}$ and $\mathrm{M}_{Y}-\mathrm{H}_{a}$ loops (left) and $\mathrm{M}_{X}-\mathrm{H}_{e}, x$ and $\mathrm{M}_{\mathrm{y}}-\mathrm{H}_{\mathrm{e}, \mathrm{x}}$ loops (right) of sVHS co-gamma media for $\Omega=60^{\circ}$.
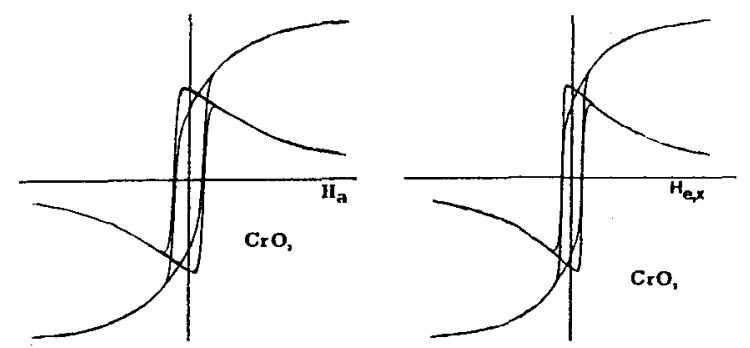

Fig. $3 \quad \mathrm{M}_{x}-\mathrm{H}_{a}$ and $\mathrm{M}_{y}-\mathrm{H}_{a}$ loops (left) and $M_{X}-H_{e}, x$ and $\mathrm{M}_{\mathrm{Y}}-\mathrm{H}_{\mathrm{e}}, \mathrm{x}$ loops (right) of advanced $\mathrm{CrO}_{2}$ media for $\Omega=$ $60^{\circ}$.
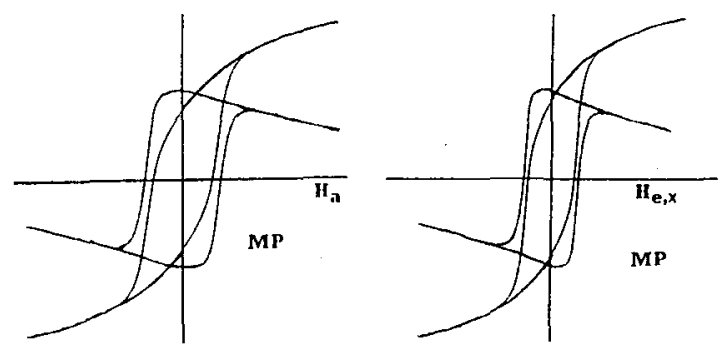

Fig. $4 \quad \mathrm{M}_{X}-\mathrm{H}_{\mathrm{a}}$ and $\mathrm{M}_{\mathbf{y}}-\mathrm{H}_{\mathrm{a}}$ loops (left) and $M_{x}-H_{e}, x$ and $\mathrm{M}_{\mathrm{Y}}-\mathrm{H}_{\mathrm{e}} \mathrm{X}$ loops (right) of $8 \mathrm{~mm}$ MP media for $\Omega=60^{\circ}$.

The isotropic Ba-ferrite media shown in Figure 6 have a smaller $M_{y}$ component, while the perpendicularly oriented Ba-ferrite (Figure 7) and the $\mathrm{CoCr}$ (Figure 8) media have a small and distinctly different $M_{y}$ curve.

Using the above transformations, we calculated the intrinsic 

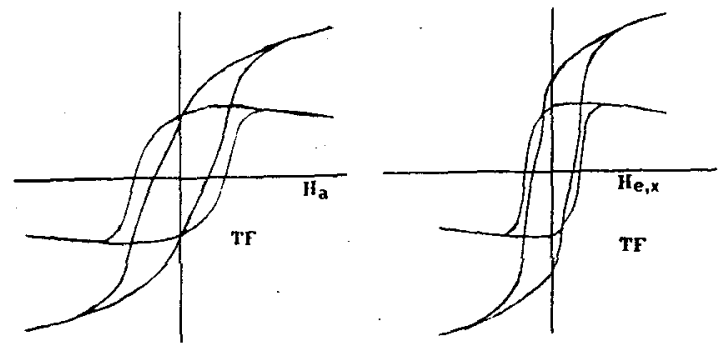

Fig. $5 \quad M_{X}-H_{a}$ and $M_{Y}-H_{a}$ loops (left) and $M_{x}-H_{e}, x$ and $\mathrm{M}_{\mathrm{Y}}-\mathrm{H}_{\mathrm{e}} \times$ loops ( $\mathrm{right}$ ) of $8 \mathrm{~mm}$ thin film media for $\Omega=60^{\circ}$.
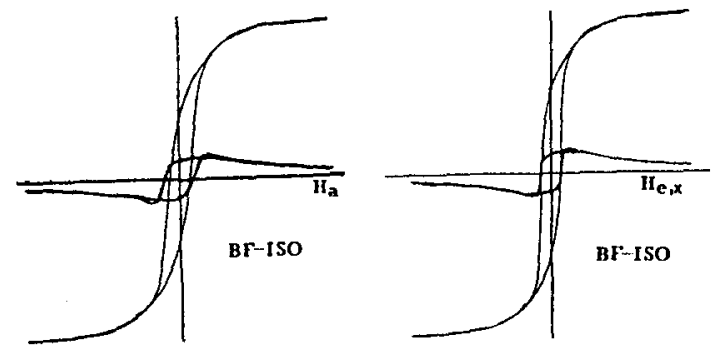

Fig. $6 \quad M_{X}-H_{a}$ and $M_{y}-H_{a}$ loops (left) and $M_{X}-H_{e, x}$ and $\mathrm{M}_{Y}-\mathrm{H}_{\mathrm{e}}, \mathrm{x}$ loops (right) of isotropic Ba-ferrite floppy disk media for $\Omega=60^{\circ}$.
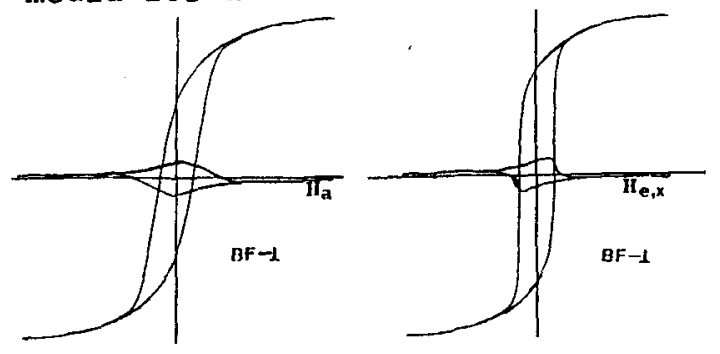

Fig. $7 \quad \mathrm{M}_{\mathrm{x}}-\mathrm{H}_{\mathrm{a}}$ and $\mathrm{M}_{\mathrm{y}}-\mathrm{H}_{\mathrm{a}}$

loops (left) and $\mathrm{M}_{x}-\mathrm{H}_{\mathrm{e}} \mathrm{x}$ and $\mathrm{M}_{\mathrm{Y}}-\mathrm{H}_{\mathrm{e}, \mathrm{x}}$ loops ( $\mathrm{r}$ ight) of perpendicularly oriented Ba-ferrite tape media for $\Omega=60^{\circ}$.

angular dependence $\mathrm{H}_{\mathrm{C}}(\Omega)$ for the various media, and the results are shown in Figures 9-15, along with the uncorrected values of $\mathrm{H}_{\mathrm{C}}$. We observe that the SVHS tape and the Co-Ni-P longitudinal thin film have a similar $\mathrm{H}_{\mathbf{C}}(\Omega)$ dependence
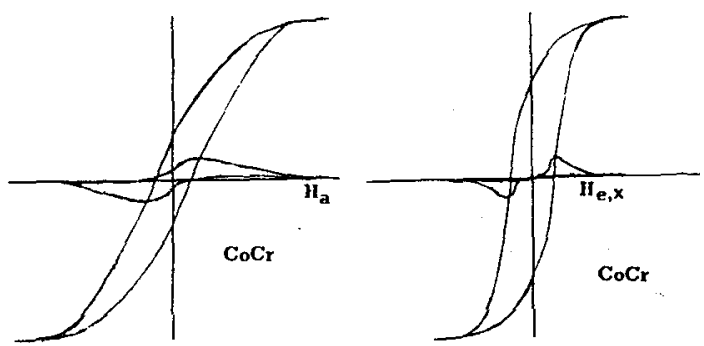

Fig. $8 \quad \mathrm{M}_{\mathrm{X}}-\mathrm{H}_{\mathrm{a}}$ and $\mathrm{M}_{\mathrm{Y}}-\mathrm{H}_{\mathrm{a}}$ loops (left) and $M_{x}-H_{e}$, and $\mathrm{M}_{\mathrm{y}}-\mathrm{H}_{\mathrm{e}, \mathrm{x}}$ loops ( $\mathrm{right}$ ) of $\mathrm{CoCr}$ perpendicular anisotropy media for $\Omega=60^{\circ}$.

decreasing monotonically with $\Omega$. on the other hand, the $\mathrm{CrO}_{2}$ and the MP tapes have a very different $\mathrm{H}_{\mathrm{C}}(\Omega)$ dependence, exhibiting a slight peak at about $60^{\circ}$, which is reminiscent of a curling mode of magnetization reversal [ ]. The isotropic Ba-ferrite has its intrinsic $\mathrm{H}_{C}$ essentially independent of $\Omega$, as we would expect. In all of these cases, the corrected $\mathrm{H}_{\mathbf{C}}$ is lower than the uncorrected $\mathrm{H}_{\mathrm{C}}$ at all values of $\Omega$, except for $0 \circ$ and $90^{\circ}$ where the two are equal. Figures 14 and

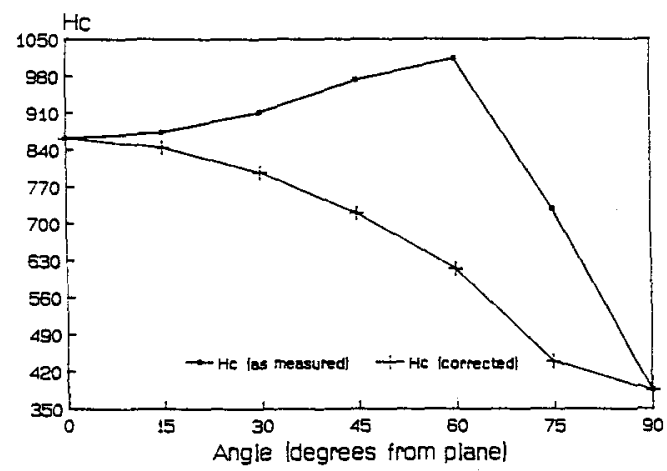

Fig. 9 Angular variation of $\mathrm{H}_{\mathrm{C}}$ of SVHS media before and after demagnetization correction.

15 show the corresponding results for the perpendicularly oriented media (Ba-ferrite and $\mathrm{CoCr}$ ). In this case, the corrected $\mathrm{H}_{C}$ is 


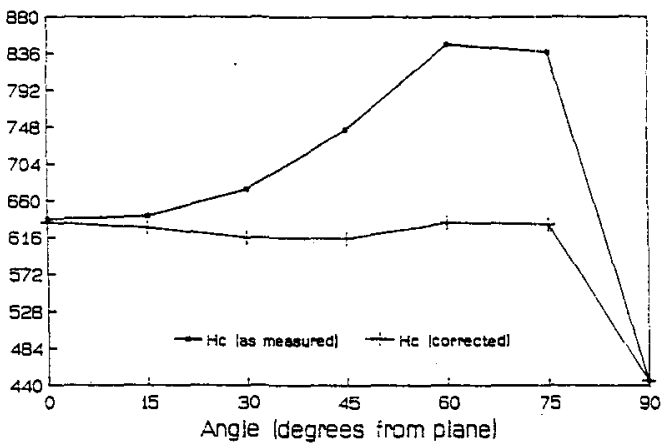

Fig. 10 Angular variation of $\mathrm{H}_{\mathrm{C}}$ of $\mathrm{CrO}_{2}$ media before and after demagnetization correction.

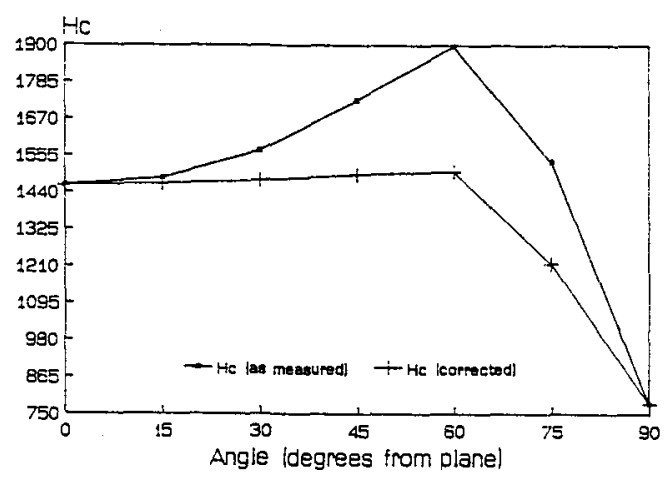

Fig. 11 Angular variation of $\mathrm{H}_{\mathrm{C}}$ of $8 \mathrm{~mm}$ MP media before and after demagnetization correction.

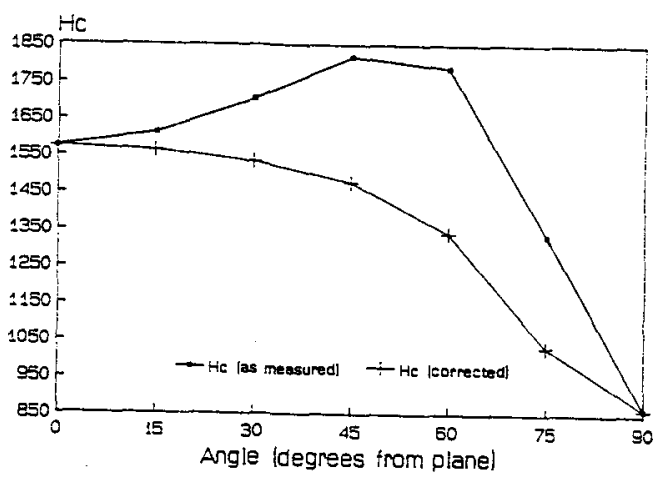

Fig. 12 Angular variation of $\mathrm{H}_{\mathrm{C}}$ of $8 \mathrm{~mm}$ thin film media before and after demagnetization correction.

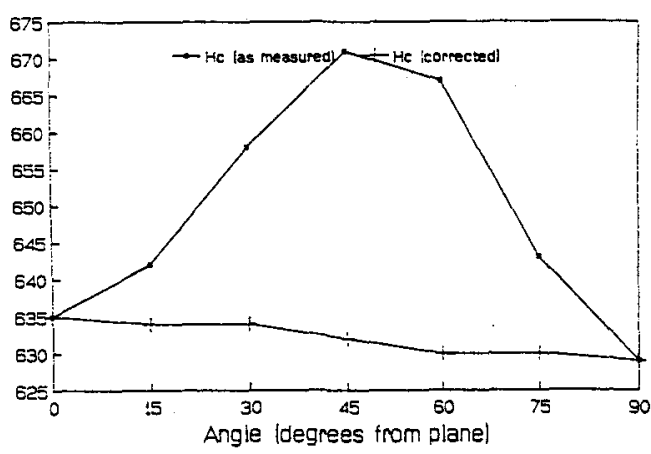

Fig. 13 Angular variation of $\mathrm{H}_{C}$ of isotropic Ba-ferrite floppy disk media before and after demagnetization correction.

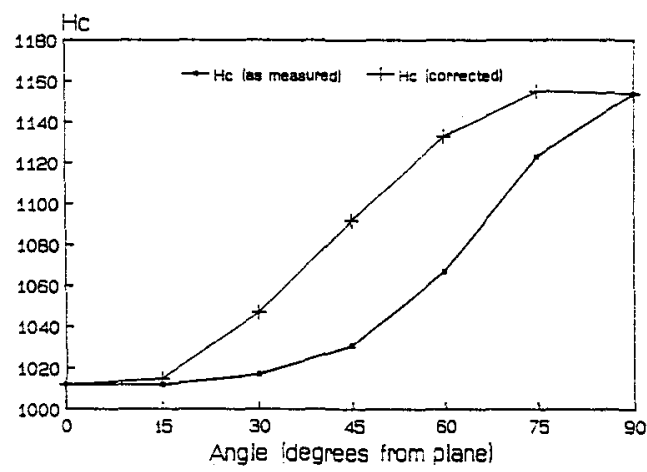

Fig. 14 Angular variation of $\mathrm{H}_{\mathrm{C}}$ of perpendicularly oriented Ba-ferrite tape media before and after demagnetization correction.

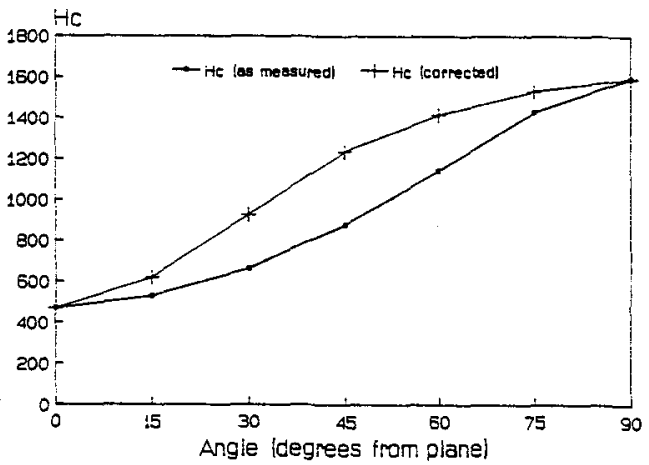

Fig. 15 Angular variation of $\mathrm{H}_{\mathrm{C}}$ of $\mathrm{CoCr}$ perpendicular anisotropy media before and after demagnetization correction. 
higher than the uncorrected $\mathrm{H}_{C}$. Otherwise, the shape of the intrinsic $\mathrm{H}_{C}(\Omega)$ curves resembles that of the SVHS and Co-Ni-P, except for the $90^{\circ}$ angular displacement of the perpendicular anisotropy media.

To draw some conclusions regarding the mechanism of magnetization reversal in these media, it is necessary to normalize the $\mathrm{H}_{\mathrm{C}}(\Omega)$ curves to the average anisotropy field $H_{k}$. This will form the subject of a subsequent publication.

\section{REFERENCES}

[1] S. Strikman and D. Treves, Jour. de Phys, et Rad., Vol. 20,286 (1959).

[2] K. Ouchi and S. Iwasaki, IEEE Trans. Mag., Vol. 23, 2443-2448, september 1987.

[3] S. Iwasaki and Y. Nakamura, IEEE Trans. Mag., Vol. 13, 1272-1277, september 1977.

[4] T. Fujiwara, IEEE Trans. Mag., Vol. 23, 3125-3130, September 1987.

[5] K. Ouchi and $S$. Iwasaki, IEEE Trans. Mag., Vol. 24, 3009-3011, November 1988 . 